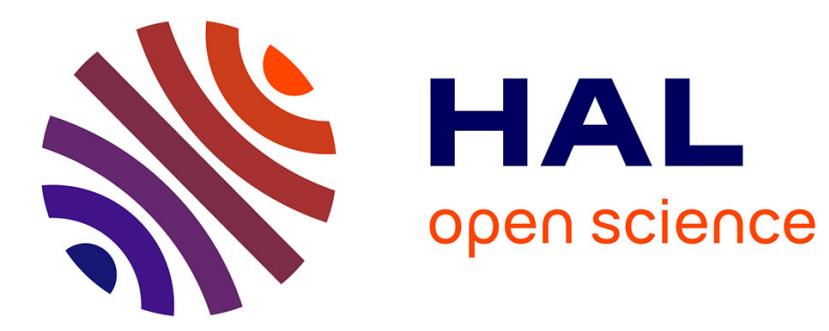

\title{
Retour sur un dogmatisme peu ordinaire Elise Marrou
}

\section{To cite this version:}

Elise Marrou. Retour sur un dogmatisme peu ordinaire. Raison-publique.fr : arts, politique, société, 2016, 10.3917/rpub.020.0099 . hal-02504986

\section{HAL Id: hal-02504986 https://hal.science/hal-02504986}

Submitted on 11 Mar 2020

HAL is a multi-disciplinary open access archive for the deposit and dissemination of scientific research documents, whether they are published or not. The documents may come from teaching and research institutions in France or abroad, or from public or private research centers.
L'archive ouverte pluridisciplinaire HAL, est destinée au dépôt et à la diffusion de documents scientifiques de niveau recherche, publiés ou non, émanant des établissements d'enseignement et de recherche français ou étrangers, des laboratoires publics ou privés. 


\title{
RETOUR SUR UN DOGMATISME PEU ORDINAIRE
}

\author{
Élise Marrou
}

Presses universitaires de Rennes | «Raison publique »

2016/1 N²0 | pages 99 à 111

ISSN 1767-0543

ISBN 9782753548770

Article disponible en ligne à l'adresse :

https://www.cairn.info/revue-raison-publique1-2016-1-page-99.htm

Distribution électronique Cairn.info pour Presses universitaires de Rennes.

(C) Presses universitaires de Rennes. Tous droits réservés pour tous pays.

La reproduction ou représentation de cet article, notamment par photocopie, n'est autorisée que dans les limites des conditions générales d'utilisation du site ou, le cas échéant, des conditions générales de la licence souscrite par votre établissement. Toute autre reproduction ou représentation, en tout ou partie, sous quelque forme et de quelque manière que ce soit, est interdite sauf accord préalable et écrit de l'éditeur, en dehors des cas prévus par la législation en vigueur en France. Il est précisé que son stockage dans une base de données est également interdit. 


\title{
RETOUR SUR UN DOGMATISME PEU ORDINAIRE
}

\author{
Elise MARROU ${ }^{1}$
}

C'est comme si ce jeu auquel se livre Austin autour de l'idée de péché originel en faisant un

lien de sa propre voix entre un jardin perdu et les exemples berkeleyens de la pomme et de l'arbre non perçu recevaient dans les Recherches philosophiques une expression philosophiquement sérieuse (séculière): les locuteurs humains sont toujours tourmentés par les possibilités qu'ils n'ont pas saisies, toujours abattus lorsqu'ils les saisissent ${ }^{2}$.

Depuis son émergence, la philosophie du langage ordinaire ${ }^{3}$ n'a cessé d'être soupçonnée de rendre l'enquête philosophique triviale, comme si les usages ordinaires sur lesquels elle travaille et qui sont son matériau propre contaminaient par avance ses procédures et la condamnaient à n'être qu'une entreprise de destruction de " tout ce qui est grand et important " selon l'expression de Wittgenstein au $₫ 118$ des Recherches. L'irritation qu'elle a suscitée et l'agacement qu'elle continue de provoquer ne doivent pas être pudiquement passés sous silence. Il importe au contraire de les affronter aussi directement que possible et de réfléchir aux enjeux de cette mise en cause.

En effet, on ignore trop souvent que, loin d'avoir été sourde à cette objection de principe, la philosophie du langage ordinaire en a fait un problème qui l'a conduite à examiner de plus près les motivations de sa rupture avec les méthodes philosophiques traditionnelles. C'est donc la transformation de cette objection

1. Ancienne élève de l'ENS (A/L 1996), agrégée et docteure en philosophie (Paris 1), chercheuse associée aux Archives Husserl, Elise Marrou enseigne la philosophie contemporaine et l'histoire de la philosophie à l'université Paris Sorbonne.

2. S. Cavell, Philosophie, le jour d'après demain, trad. N. Ferron, Fayard, 2011, p. 228.

3. Nous nous appuierons ici sur les œuvres de J.-L. Austin, de L. Wittgenstein et de S. Cavell. Pour une défense récente de ces procédures qui prend en charge les objections qui sont le plus couramment adressées à la philosophie du langage ordinaire, voir l'ouvrage d'A. Baz, When words are called for: A Defense of Ordinary Language Philosophy, Harvard UP, 2012. 
en une question philosophique à part entière que nous nous proposons dans le présent article de prendre pour objet. Nous le ferons en considérant les procédures des philosophes du langage ordinaire à contre-emploi, et pour ainsi dire à rebrousse-poil, en partant de ce que Stanley Cavell nomme d'une façon quelque peu surprenante leur " allure de dogmatisme »:

Je pense que cette allure de dogmatisme (air of dogmatism) est de fait présente dans ces affirmations, mais si c'est de l'intolérance, c'est parce que la tolérance pourrait seulement vouloir dire, comme c'est souvent le cas chez les intellectuels de gauche, que l'on ne prend pas au sérieux le type d'affirmation qui est en question. Après tout, c'est une affirmation concernant nos vies; si on veut parvenir à la tolérance, c'est sur les différences et les oppositions de nos vies qu'il faut la diriger ${ }^{4}$.

Dans sa conclusion aux "Problèmes esthétiques de la philosophie moderne ", Cavell réfléchit sur la rupture que les philosophes du langage ordinaire ont consommée avec la tradition. Il a d'ailleurs mis en évidence dans le chapitre qui ouvre le recueil d'essais, Dire et vouloir dire, qu'un angle d'attaque pertinent pour évaluer la nouveauté de cette manière de philosopher réside dans l'injonction à nous souvenir de " ce que nous dirions d'ordinaire dans telle ou telle situation ». Dans cet appel aux usages ordinaires présent chez Austin et chez Wittgenstein à la fois comme un recours et une tentation ${ }^{5}$, Cavell propose de voir un analogon de la "voix universelle " de la Critique de la faculté de juger. L'appel au " nous " prendrait ainsi un sens transcendantal, et la description des usages ordinaires n'aurait dès lors rien d'empirique.

Pourtant, l'auteur des Voix de la raison est le premier à reconnaître que les descriptions de ce que "nous dirions ordinairement quand " paraissent dogmatiques. Son diagnostic sur ce point ne souffre d'aucune atténuation, ni d'aucune nuance: il ne s'agit en aucun cas de dédouaner les procédures d'Austin ou de Wittgenstein de cette objection, mais de comprendre les raisons de cet air dogmatique. Cavell souligne plus précisément que l'intolérance dont les philosophies du langage ordinaire font preuve à l'égard des procédures traditionnelles exprime en réalité le sérieux de leur prétention philosophique. Or, comment assumer qu'une forme de dogmatisme (quelle qu'elle soit d'ailleurs) puisse rendre justice à la probité de l'exigence qui les guide?

Pour répondre à cette question, nous reviendrons d'abord sur le sens particulier du dogmatisme des philosophies du langage ordinaire: plutôt que d'adopter une attitude conciliatrice, Cavell prend le parti de faire apparaître la divergence qui sépare les méthodes des philosophes du langage ordinaire des philosophes

4. S. Cavell, Must we mean what we say?, 1969, Harvard UP, p. 96, trad. fr. S. Laugier, et Ch. Fournier, Dire et vouloir dire, Paris, Cerf, 2009, p. 194-195.

5. Cavell emploie d'ailleurs le terme d'appeal to everyday language qui possède ce double sens. 
plus traditionnels de la façon la plus radicale que possible. L'un des résultats les plus intéressants de son analyse est que ce dogmatisme découle d'une décision qui porte sur l'objet de la philosophie elle-même. C'est ainsi un dogmatisme bien étrange qui débouche tout autant sur une critique immanente des procédures de la philosophie du langage ordinaire elle-même que sur une critique de l'objet traditionnel de l'enquête philosophique.

Nous analyserons cette décision dans un second temps en examinant ses conséquences directes sur la nature de ce que nous prétendons savoir: le dogmatisme des procédures de la philosophie du langage ordinaire répond en réalité au dogmatisme fondateur de la philosophie moderne. Selon Cavell, c'est bien sur le terrain de la définition de la connaissance que se joue la rupture des deux manières de philosopher. En nous appuyant sur les analyses de The Claim of Reason et de Disowning Knowledge, nous verrons comment Cavell construit un terrain d'entente, celui d'une disputatio, qui rétablit le dialogue rompu entre le philosophe du langage ordinaire et le philosophe de la connaissance traditionnelle, et qui contraint aussi chacun à reconnaître le poids de leurs présuppositions respectives dans la détermination ce que nous prétendons savoir, et par là, à reconnaître la part dogmatique qui guide l'enquête philosophique. Nous tenterons pour conclure de comprendre le sens de ce dernier déplacement dans l'économie du récital sceptique lui-même.

\section{I - GÉNÉALOGIE DE « L'ALLURE DOGMATIQUE »}

Le point de départ de Cavell dans Dire et vouloir dire consiste à interroger le double effet produit par l'appel à l'ordinaire: les procédures du philosophe du langage ordinaire apparaissent par contraste avec les procédures traditionnelles à la fois triviales et dogmatiques. On comprend sans peine les raisons de la trivialisation de la réflexion philosophique. Mais le dogmatisme qui leur est imputé est plus problématique.

En effet, ce dernier ne s'entend pas seulement en l'occurrence comme l'absence de remise en question des préjugés ou de certaines croyances philosophiques. Le dogmatisme n'a ici rien de local, il est en l'occurrence principiel. C'est précisément la raison pour laquelle le philosophe de la connaissance traditionnelle ne peut que rejeter de prime abord ce que le philosophe qui s'appuie sur les usages ordinaires affirme. Le dogmatisme reproché au philosophe du langage ordinaire a ceci de particulier qu'il porte sur l'ensemble de sa pratique et sur les principes qui la guident. À la source même des critiques, il y a un refus viscéral que Cavell 
n'hésite pas à décrire dans les termes d'une "oppression ${ }^{6}$ ": ce qui est insupportable, ou du moins ce qui paraît difficilement soutenable, c'est "l'idée que ce que nous disons et voulons dire ordinairement puisse avoir un contrôle direct et profond sur ce que nous pouvons dire et voulons dire en philosophie ${ }^{7}$ ». Car s'il peut paraître trivial de reconnaître une normativité aux usages ordinaires, admettre en revanche que la normativité des usages ordinaires doit dicter au philosophe les modalités selon lesquelles il doit procéder ne va nullement de soi. C'est sans doute là la source du sentiment de suffocation que Cavell dépeint avec force et qu'on aurait tort d'affadir. Se soumettre à la puissance normative des usages ordinaires, c'est s'obliger à prendre position sur la nature de la situation mise en jeu:

Nous avons besoin de nous souvenir de ce que nous devrions dire et quand. Mais à quoi sert-il de nous souvenir de cela? Quand le philosophe demande: "Que devrions-nous dire ici? », ce que l'on veut dire c'est: "Quelle serait la chose normale à dire ici? », ou peut-être: Quelle est la chose la plus naturelle que nous pourrions dire ici? Et la question sert à ceci: y répondre est parfois la seule manière de dire - de dire aux autres et de dire à nous-mêmes - quelle est la situation ${ }^{8}$.

L'exigence de dire "quelle est la situation " traduit la nécessité des descriptions du philosophe du langage ordinaire. Plus la description est fine et adéquate, plus elle mord sur le réel au point que le propos du philosophe de la connaissance traditionnel en devient verbal:

En conséquence, l'une des formes prises par [les] recherches [d'Austin] est de rejeter les distinctions que l'on rencontre dans les parages de la philosophie, de les mettre hors de possession, pour ainsi dire, en en montrant de meilleures. Et meilleures non seulement parce qu'elles sont plus fines, mais parce qu'elles sont plus massives qu'elles ont, si je puis m'exprimer ainsi, un plus grand poids naturel; qu'elles apparaissent normales et même inévitables quand les autres sont sinistrement arbitraires; utiles quand les autres semblent forcées; réelles quand les autres sont académiques; fécondes quand les autres restent sur place. L'intention positive dans les distinctions d'Austin ressemble à l'intention du critique d'art quand il compare et distingue des œuvres d'art, à savoir que sous cet éclairage croisé, les capacités et le relief individuel de l'objet en cause sont amenés à notre attention et placés en leur point de lisibilitép.

On a parfois l'impression que les différences chez Austin pénètrent les phénomènes qu'elles relatent et une fois que l'on ressent cette impression, ce sera le philosophe de la connaissance traditionnelle qui nous semblera ne parler que sur des mots ${ }^{10}$.

L'appel à l'ordinaire n'a rien d'une simple question de mots, car les distinctions conceptuelles mises en évidence par la description des usages ordinaires ne

6. Dire et vouloir dire, "Devons-nous vouloir dire ce que nous disons? ", op. cit., p. 73.

7. Ibid.

8. Dire et vouloir dire, "Devons-nous vouloir dire ce que nous disons? ", op. cit., p. 99.

9. Dire et vouloir dire, "Austin critique ", op. cit., p. 204-5.

10. Ibid. 
sont autres que les articulations du réel. D’où la caractérisation par Austin de son projet comme d'une "phénoménologie linguistique ${ }^{11}$ ". L'appel à l'ordinaire n'est pas un appel aux cas empiriques, concrets, positifs. Dans Sense and Sensibilia, Austin reproche d'ailleurs à la lignée de la tradition empiriste britannique son attitude "scolastique " qui ne prend en considération que des faits imparfaitement étudiés ${ }^{12}$. La description n'est pas tant une description concrète et empirique que la description de ce qui est en tant que ce qui est nous importe. Elle ne produit donc pas une description du monde, ni de notre ordinaire, mais une révélation du monde ${ }^{13}$. Selon Austin, il n'y a pas d'autre manière de faire l'expérience de cette prise sur le monde qu'est notre connaissance que de passer par cette description des usages. Les descriptions grammaticales ne nous donnent pourtant pas un sens plus aiguisé du réel au sens où elles porteraient à notre connaissance de nouveaux faits. Elles révèlent plutôt les conditions de ce que nous savons, dans les termes de Cavell, elles révèlent nos critères. Lorsque le philosophe du langage ordinaire se demande ce que nous devrions dire, "what we should say?", il ne se demande pas "what we ought to say". Travailler à produire des contextes dans lequel un concept ou une expression ont un sens, c'est travailler à produire l'espace adéquat dans lequel ce concept ou cette expression sont opératoires. La description ne résulte pas d'un rabaissement des prétentions philosophiques, mais d'un travail d'exemplification qui produit « l'espace exact dans lequel elles peuvent se déplacer sans avoir d'effet corrupteur»:

Mais le philosophe qui part du langage ordinaire se préoccupe moins de dénoncer et de punir des crimes odieux contre l'intellect que de compenser, au civil, les dommages qu'il a subis; de rétablir tout défaut d'équilibre, toute minime usurpation, dans l'esprit. Ceci demande inévitablement de réintroduire des idées qui sont devenues tyranniques (par exemple, l'existence, l'obligation, la certitude, l'identité, la réalité, la vérité...) dans les contextes spécifiques de leur fonctionnement naturel. Il ne s'agit pas ici de retailler des idées trop grandes pour leur faire retrouver leur format adéquat, mais de leur donner l'espace exact dans lequel elles peuvent se déplacer sans avoir d'effet corrupteur ${ }^{14}$.

La réappropriation par Cavell des procédures du philosophe du langage ordinaire revient par conséquent à distinguer deux variétés de dogmatisme. La première est constitutive de la provocation du philosophe du langage ordinaire et elle doit être maintenue. Elle se confond avec l'exigence réaliste que nous venons de rappeler brièvement. Nous devons nous défaire de la seconde qui caricature la motivation

11. J. -L. Austin, Écrits philosophiques, « Plaidoyer pour les excuses ", Paris, Le Seuil, 1994, p. 144.

12. Sense and Sensibilia, London, Oxford UP, 1964, p. 3 (Le Langage de la perception, Paris, Vrin, 2007, p. 79).

13. Dire et vouloir dire, "Austin critique ", op. cit., p. 206.

14. Dire et vouloir dire, "Devons-nous vouloir dire ce que nous disons ", op. cit., p. 95 (nous soulignons). 
de l'enquête philosophique traditionnelle. Examinons de plus près ces deux sens opposés du dogmatisme mis en jeu par les procédures d'Austin.

1. Le dogmatisme est d'abord lié à l'usage de la première personne du pluriel, $\mathrm{du}$ « nous » qui n'est pas ici seulement postulé, mais que le philosophe du langage ordinaire s'est toujours déjà arrogé. Ce dogmatisme que nous n'avons d'autre choix que d'assumer et que le philosophe traditionnel récuse réside dans la prétention du philosophe du langage ordinaire à parler au nom des autres. Cette dimension est constitutive des procédures du langage ordinaire. Dans Un ton pour la philosophie, Cavell voit ainsi dans « la prétention systématique de la voix ou l'arrogante assomption du droit à parler pour les autres ${ }^{15}$ » la source même de la manière de philosopher du second Wittgenstein et d'Austin. Les analyses de 104 Benveniste sur les spécificités du pronom " nous " pourraient ici prolonger celles de Cavell: le « je » qui dit « nous » ne se décentre donc pas, il s'élargit pour englober un ensemble de non-je. "Le " nous » annexe au " je " une globalité indistincte d'autres personnes». La subjectivité singulière qui dit "je » reste le centre de l'énonciation pour proclamer ou proférer le «nous». Que le philosophe du langage ordinaire parle au nom de la communauté des êtres qui partagent ces critères montre qu'on ne peut pas séparer pour Cavell la dimension épistémologique de l'enquête du sens moral et politique de la description. Ce dogmatisme n'a pas à être supprimé (comment serait-ce d'ailleurs possible?), il doit être maintenu et assumé sous peine de ne pouvoir mener à bien quelque description que ce soit. En revanche, il n'est évidemment pas à l'abri des mises en doute sceptiques qui révèlent la vulnérabilité et la fragilité de cette invocation sûre d'elle-même dont le fondement n'est autre que les accords communs et partagés; en un sens - et c'est sans doute l'aspect le plus intéressant de l'analyse de Cavell - cette allure dogmatique est elle-même provoquée par le doute.

2. En revanche, en analysant l'accumulation des termes critiques d'Austin à l'égard de la philosophie traditionnelle, Cavell montre qu'Austin marginalise ses propres procédures. L'auteur du " Plaidoyer pour les excuses" ne rend pas justice à la radicalité de sa propre méthode. Cavell y voit un "manque de sa philosophie " et même "un échec de son enseignement ${ }^{16}$ ". Cette critique ne revient pas seulement à souligner l'écart entre ce que Austin dit qu'il fait et ce qu'il fait véritablement. Ce n'est pas non plus seulement que les procédures aus-

15. Voir le premier essai d'Un ton pour la philosophie, tr. É. Domenach et S. Laugier, Bayard, 1994.

16. Dire et vouloir dire, op. cit., p. 67 
tiniennes seraient trop constituantes et pas assez sceptiques (ou encore qu'elles ne rendraient compte que de la force et non de la vulnérabilité des critères qui régissent les usages). Austin ne manque pas seulement la dimension sceptique de l'ordinaire selon Cavell: en caricaturant les procédures traditionnelles, il se rend incapable de comprendre le problème philosophique dont elles sont l'expression. C'est bien là que réside la part de dogmatisme dans sa méthode que Cavell rejette entièrement et dont l'enseignement de Wittgenstein doit nous défaire ${ }^{17}$. C'est en un sens l'analyse critique de ce dogmatisme qui donne son impulsion à l'enquête des Voix de la raison.

En effet, à décrire les procédures de la philosophie du langage ordinaire dans les termes d'Austin, on ne peut pas rendre compte du sentiment de la découverte caractéristique de l'enquête philosophique traditionnelle. C'est donc bien une insatisfaction à l'égard de la description qu'Austin propose de ses propres méthodes qui conduit Cavell à formuler un terrain de la confrontation des deux méthodes. Telle que nous comprenons la progression de la seconde partie des Voix de la raison, l'enjeu revient donc en partie à mettre en évidence les insuffisances des procédures de la philosophie du langage ordinaire d'Austin à partir du prisme de la singularité des questions posées par le philosophe de la connaissance traditionnelle. Pour ce faire, Cavell reconstruit patiemment le dialogue qui était à la fois rompu par le philosophe du langage traditionnel et par le philosophe du langage ordinaire. Il élabore pour l'un et l'autre, pourrait-on dire, le terrain d'entente pour construire une disputatio. Il s'agit donc de:

- faire entendre au philosophe du langage ordinaire que la question "originante " du philosophe de la connaissance traditionnelle qui vise à rendre raison de la connaissance dans son ensemble est bel et bien réelle.

- faire entendre au philosophe de la connaissance traditionnelle que sa question, bien que suscitée par une demande réelle, ne peut pas déboucher sur une réponse, du moins s'il entend par là une justification et une fondation de l'ensemble de nos prétentions épistémiques.

Il est donc crucial pour notre propos que ce face à face confronte en réalité deux formes de dogmatismes qui selon Cavell sont aussi préjudiciables l'une que l'autre:

Le problème reste donc entier: pourquoi le philosophe soulève-t-il à propos d'un objet générique, une question, alors même que les circonstances sont telles qu'il n'y a normalement pas à douter que l'objet soit là? Si j'insiste sur le fait qu'il y a là bel et bien un problème, c'est à la

17. Pour le montrer d'une façon plus circonstanciée, il faudrait s'appuyer aussi bien sur "Austin critique " ("Austin at criticism») dans Dire et vouloir dire, que sur la $2^{\mathrm{e}}$ partie des Voix de la raison en revenant sur l'opposition des critères austiniens et des critères wittgensteiniens, synthétisée de façon limpide dans « L'événement wittgensteinien ", Philosophie: le jour d'après demain, op. cit., p. 209-230. 
fois contre le philosophe du langage ordinaire et contre le philosophe dit classique. Car on peut imaginer que le premier dira: "Il est tout simplement évident que la question ne se pose pas; le philosophe s'obstine à la poser par pur entêtement ou par aveuglement. » Mais il s'agit là d'une considération entièrement dogmatique, et qui n'a rien de convaincant, à la différence d'autres analyses du langage ordinaire à propos de situations ordinaires. Inversement le philosophe traditionnel présentera, on peut s'y attendre, une défense du genre: «Il est tout à fait évident qu'il faut poser la question: peu importe qu'il s'agisse d'une question qu'on ne se pose habituellement pas. Au contraire qu'il faille la poser montre bien la complaisance du sens commun, l'inadéquation du langage ordinaire. " Réplique malheureusement tout aussi dogmatique que la position contre laquelle elle se défend. Le philosophe doit en effet bien mesurer que la raison pour laquelle il lui "faut" soulever cette question serait admise par quiconque comprendrait ce qu'il dit. Ses questions sont parfaitement ordinaires (" est-ce que ce ne pourrait pas être de la cire? ", "Le voyez-vous en totalité? "); et il connaît aussi bien que quiconque, les circonstances où l'on pose ordinairement ces questions, ainsi que la portée des réponses qu'on leur donne (" nous ne le voyons pas en totalité, nous ne savons donc pas que c'en est un ».), - l'ensemble de sa procédure reposant en fait sur une telle connaissance. Toute critique des investigations traditionnelles de la connaissance qui se veut fondée sur une attention à « ce qui est dit ordinairement quand...» doit rendre compte du fait que les philosophes classiques - lesquels maîtrisent tout de même le langage qu'ils écrivent - ont admis à propos d'une question, qu'elle requiert une réponse qu'ils auraient eux-mêmes, dans d'autres circonstances (à l'image de "l'homme ordinaire ") écartée comme absurde ${ }^{18}$.

Et d'une manière encore plus ramassée:

Ce que je suis donc en train de demander, c'est que nous prenions la question originelle - et originante - du philosophe avec le même sérieux que le philosophe du langage ordinaire souhaite que nous prenions tout énoncé formulé par un être humain. (C'est ce souhait et la fidélité de son expression qui représentent l'une des valeurs permanentes de ce motif et de cette " méthode " d'activité philosophique) ${ }^{19}$.

Il est désormais clair que la réappropriation que Cavell propose des méthodes d'Austin est fondée sur une critique immanente de ses procédures. D'une façon tout à fait explicite, Cavell fait jouer les descriptions grammaticales de Wittgenstein contre les termes critiques d'Austin pour montrer que seules les premières provoquent selon l'expression des Recherches « une rotation de perspective autour du point fixe de notre besoin véritable » $(\$ 108)$. Or, cette transformation n'est possible selon Cavell quà condition de comprendre ce qui pousse le philosophe de la connaissance à sortir des cadres qui lui ont été impartis, c'est-à-dire qu'à condition d'être tout autant un philosophe du «langage métaphysique ${ }^{20}$ " que du langage

18. Les Voix de raison, op. cit., p. 103-104.

19. Les Voix de la raison, op. cit., p. 217.

20. Sur cette suggestion d'un Wittgenstein philosophe du langage métaphysique, voir " Lévénement wittgensteinien " dans Philosophie, le jour d'après demain, p. 212. "L'événement-Wittgenstein » nous paraît d'ailleurs plus fidèle à l'inspiration nietzschéenne revendiquée par Cavell dans ce texte. Voir également dans Un ton pour la philosophie, op. cit., p. 32. 
ordinaire. La détermination que le philosophe de la connaissance traditionnelle propose du concept de "savoir » doit être comprise si l'on entend laisser place à un concept de certitude qui ne soit plus désengagé et qui ne laisse plus de côté « tout ce qui touche à l'apprentissage et à l'acquisition du savoir ».

\section{II - RÉENGAGER LES PROCÉDURES ÉPISTÉMOLOGIQUES DANS LES PRATIQUES HUMAINES:}

"La morale pour la connaissance prise dans son ensemble » qui résulte de la confrontation des deux formes de dogmatisme consiste à surmonter les présuppositions du philosophe de la connaissance traditionnelle et celles du philosophe du langage ordinaire. Or, la divergence des objets de l'enquête de part et d'autre recoupe exactement la divergence sur le sens du savoir qui est mobilisé. Quels sont les faits à partir lesquels la philosophie doit mener son enquête? Quel est le donné considéré de part et d'autre comme le donné légitime du philosophe? Pour le dire tout d'abord d'une façon très générale, Austin reproche à la philosophie traditionnelle de "travailler sur de petits exemples ridicules, arbitraires, qui dévalorisent les investigations dès leur commencement ${ }^{21} »$. En effet ses objets sont " des bouts de cire, des tables, des chaises, des maisons, des hommes, des enveloppes, des cloches, des feuilles de papier, des tomates, des tableaux noirs, des crayons, etc. ", c'est-à-dire des objets qui ne posent aucun problème de reconnaissance, de récognition, de ré-identification, des objets que Cavell nomme pour les besoins de sa propre cause, des objets génériques:

Mais supposons que dans ce domaine, les théoriciens classiques de la connaissance n'aient pas eu le choix et que leur obsession ne fasse en réalité que suivre et révéler la nature de la question qui les obsède. Tous leurs objets ont en effet quelque chose de commun: ce sont très spécifiquement des objets qui ne posent pas de problèmes de recognition, d'identification ou de description; le seul problème à leur sujet serait, à la rigueur, non pas de dire ce qu'ils sont mais le problème de la possibilité ou de l'impossibilité de savoir s’ils existent, s'ils sont réels, s'ils sont effectivement là. Je nommerai parfois ces objets dans une intention heuristique " objets génériques " par opposition aux objets qui constituent les exemples d'Austin, que j'appellerai " objets spécifiques". Par ces dénominations je ne cherche pas à suggérer qu’il y aurait deux genres d'objets dans le monde, mais plutôt à résumer l'esprit dans lequel un objet est mis en discussion, la sorte de problèmes qui sont posés à son sujet et la manière dont il concentre sur lui l'essentiel de l'investigation ${ }^{22}$.

Cavell précise que la distinction entre l'objet spécifique exemplifié par le chardonneret de Austin dans Other Minds et l'objet générique représenté par les sense

21. Les Voix de la raison, op. cit., p. 96.

22. Les Voix de la raison, op. cit., p. 97. 
data indubitables de rougeur et de rondeur de H. H. Price perçus à partir de la tomate dans Perception n'est formulée que par commodité en termes d'objets. Cavell nous fournit ici un outil qui permet de distinguer l'esprit dans lequel la scène de la connaissance s'édifie et par là, l'esprit dans lequel le problème de la connaissance est posé. C'est ce qu'il nomme pour sa part « the explicit stage-setting of the investigation". Au fond, la distinction entre l'objet générique et l'objet spécifique fait s'affronter deux choix opposés présentés comme une lecture non biaisée de l'expérience perceptuelle, alors que chacun exprime une reconstruction théorique de l'expérience lourdes de présupposés épistémologiques.

Sur le versant de la philosophie de la connaissance traditionnelle, l'enquête trouve son origine dans les Méditations de Descartes. On ne pourra rendre compte de sa nécessité qu’à la condition de prendre au sérieux l'idée même que le scep108 tique a bien découvert un fait qui porte sur la connaissance dans son ensemble. Nous ne pouvons reprendre ici chacune des étapes de la démonstration de Cavell dans la seconde partie des Voix de la raison. Nous nous contenterons donc d'extraire ce qui nous permet de caractériser de manière plus précise le dogmatisme mis en jeu:

1. Le premier présupposé qui définit la tradition cartésienne dont H. H. Price par exemple hérite dans Perception (et qui permet à Cavell de reconstruire le récital sceptique) est fondationnaliste. La philosophie de la connaissance traditionnelle postule que sans un cas paradigmatique de certitude (best case for knowledge) - on passe ainsi du Cogito à certaines expériences privilégiées en matière de vue et de toucher érigées en fondement - nous ne pourrions pas entretenir une quelconque croyance concernant les choses matérielles:

Tout homme entretient un grand nombre de croyances au sujet des choses matérielles, par exemple qu'il existe une table carrée dans cette pièce. Il est évident que toutes ces croyances sont fondées sur la vue et le toucher: fondées sur eux au sens où si nous n'avions pas eu certaines expériences particulières en matière de vue et de toucher, il ne serait ni possible ni raisonnable d'entretenir ces croyances ${ }^{23}$.

Quand je vois une tomate, je puis douter de beaucoup de choses. Je puis douter si c'est une tomate que je vois, et non pas un morceau de cire habilement peint. Je puis douter qu'il y ait le moindre objet matériel devant. J'ai peut-être pris un reflet pour une tomate; je suis peut-être même victime d'une hallucination ${ }^{24}$.

23. H. H. Price, Perception, London, Methuen, 1932, $2^{e}$ éd. 1964, p. 1.

24. H. H. Price, Perception, op. cit. p. 3. 
2. Cette reconstruction de l'expérience perceptuelle est pensée par le philosophe de la connaissance traditionnelle de façon à ce que la question générale de la justification de la connaissance paraisse avoir un sens.

Ces deux traits que Stanley Cavell épingle comme définitoires du récital sceptique moderne rejoignent d'une façon significative ce que Michael Williams dans Groundless Belief comprend sous l'expression "appeal to the given " (en gardant le sens de la tentation qui s'ajoute en anglais au terme français):

En réalité, la pratique de Price trahit sa théorie. Car il n’a pas découvert les sense data simplement en regardant d'une manière non biaisée les contenus de sa conscience perceptuelle. Il a plutôt adopté le dispositif philosophique déjà sophistiqué de se placer dans une situation perceptuelle - de regarder une tomate dans les conditions normales de lumière etc. et de tenter ensuite de découvrir quelle croyance il était tenté de former dans cette situation qui ne pourrait pas être mise en doute. Il n'était pas intéressé par une simple conviction subjective: il se demandait en effet ce qui pouvait être mis en doute sur un plan théorique. Or, cette procédure est de part en part épistémologique ${ }^{25}$.

Price ne découvre pas l'attitude mentale d'acquaintance. Il sait par avance qu'elle doit exister. Car il écrit qu'il doit y avoir une sorte ou des sortes de présences à la conscience qui peuvent être nommées directes; sinon nous serions aux prises d'une régression à l'infini ${ }^{26}$.

3. Le fondationnalisme et la prétention à poser le problème de la connaissance dans son ensemble pris ensemble offrent le levier qui dégage la possibilité du scepticisme, c'est-à-dire de la découverte d'un échec de la connaissance: car, ce n'est que si la base infaillible et indubitable de notre savoir devient sujette au doute qu'un diagnostic sur la fragilité de notre connaissance dans son ensemble pourra être formulé. Là encore, les analyses de Stanley Cavell et de Michael Williams convergent de façon remarquable, car l'opération sceptique est indexée sur le passage de la remise en question d'une prétention particulière à l'adoption du doute porté sur la prétention à savoir quoi que ce soit. Le diagnostic est porté sur l'expérience de la découverte dont le scepticisme est la traduction. Mais ils ne s'en tiennent pas là: ils montrent tous deux pour quelles raisons (contextuelles) le doute ne peut avoir la portée universelle que le sceptique lui confère. C'est en effet ce passage de la validité particulière d'une prétention à savoir à son invalidité universelle qui est mise en cause dans le "non-claim context" par Cavell ${ }^{27}$ et par le diagnostic théorique que Michael Williams porte sur le scepticisme radical ${ }^{28}$.

25. M. Williams, Groundless Belief, Princeton UP, p. 44 (nous traduisons).

26. M. Williams, Groundless Belief, Princeton UP, p. 45 (nous traduisons).

27. Les Voix de la raison, op. cit., p. 326 et suiv.

28. Dans Groundless Belief et dans Unnatural Doubts, Princeton UP, 1996. 
Cette reconstruction de l'expérience perceptuelle conduit à réinterroger les données propres à la formulation de l'enquête moderne sur la connaissance dont résulte une épistémologie qui vise à déterminer les conditions de possibilité et de validité générales de la connaissance. La scène moderne de la connaissance telle qu'elle a été configurée par Descartes et dont la tradition empiriste anglo-saxonne a hérité élève pour la première fois l'existence du monde extérieur au rang de problème. Or, elle ne peut le faire que parce qu'elle s'appuie sur un concept de connaissance qui s'est désengagé de son ancrage dans l'apprentissage et l'entraînement des pratiques pour s'arrimer au seul concept de certitude cartésienne. Le diagnostic porté sur la morale de la connaissance dans son ensemble est bien un diagnostic sur la portée des présuppositions sur le savoir qu'a entérinées la modernité:

Si l'on met de côté l'époque actuelle où l'épistémologie subit de l'intérieur une mise en question radicale, cette tradition qui remonte à Descartes et à Locke veut que le concept de connaissance soit disjoint de ce qui touche à l'information, à l'apprentissage ou à l'acquisition du savoir et arrimé au seul concept de certitude, en particulier à une certitude dérivée des (de mes) sens. À un stade relativement précoce des enquêtes épistémologiques, le monde dont on constate normalement la présence (le monde en l'existence duquel on " croit " pour reprendre la formulation typique) est mis en question et s'évanouit; et l'on s'aperçoit que toute relation à un monde quelconque suppose que celui-ci soit, comme on dit, "présent aux sens "; d'où notre désarroi à constater que ce monde-là n'est précisément pas le monde tel que nous l'entendons. C'est alors que celui qui doute se laisse entraîner au scepticisme, puisque l'existence du monde extérieur devient pour lui un problème ${ }^{29}$.

À mon avis, l'une des raisons en est que l'exploration de la connaissance de soi a été si étonnamment peu tentée, lorsque Bacon, Locke et Descartes préparaient l'établissement d'une nouvelle science. L'épistémologie classique s'est concentrée sur la connaissance des objets (et bien sûr des mathématiques), et non pas sur la connaissance des personnes. C'est certainement l'un des faits les plus frappants de la philosophie moderne considérée dans sa totalité et on ne comprend pas son histoire tant qu'on ne rendra pas compte de ce fait ${ }^{30}$.

La réduction de la connaissance à la certitude et l'abstraction de ses procédures de ce que Cavell appelle " matters of information, skill and learning" est solidaire de la réduction de la connaissance à ce que je connais, et même plus précisément à ce que j'expérimente dans une compréhension empiriste de l'expérience. Le fondement égologique déboucherait ainsi curieusement sur une occultation de la connaissance de soi. C'est pourquoi Cavell propose, selon l'expression de James Conant, une détermination «inclusive» du scepticisme selon laquelle est sceptique toute conception qui tient l'existence pour un problème de connaissance:

C'est dire que je ne vais pas me limiter, dans l'emploi du terme [de scepticisme] aux philosophies qui nient que nous puissions en fin de compte jamais savoir; j'étends ce qualificatif à

29. Disowning Knowledge, 1987, Cambridge UP, p. 94, trad. fr., p. 150-1.

30. Les Voix de la raison, op. cit., p. 68 
toute conception qui tient l'existence du monde pour un problème de connaissance. [...] De ce point de vue, scepticisme et dogmatisme ne se laissent pas départager ${ }^{31}$.

La réponse de la philosophie du langage ordinaire au scepticisme qui porte sur l'existence du monde extérieur n'est pas de l'ordre d'une réfutation. Elle relève bien davantage d'un diagnostic porté sur la validité du mouvement d'universalisation du doute sceptique moderne. Elle remonte ainsi à la source qui tout à la fois suscite et alimente les doutes sceptiques. Elle rend compte du fait même qu'ils ne sont ni fictifs ni gratuits. Elle est donc dès lors bien une réponse au sens strict, alors que la critique d'Austin n'opposait au scepticisme à l'égard du monde extérieur qu'une fin de non-recevoir. D’une part, les procédures de la philosophie du langage ordinaire qui ont permis de montrer que le concept de savoir est chargé des trois présupposés que nous avons relevés reposent au contraire sur l'idée qu'on ne peut pas dissocier le savoir de tout ce qui touche à «l'information, à l'apprentissage ou l'acquisition ». Elles s'appuient donc à leur tour sur un concept de savoir qui présentent en réalité tous les traits du concept de certitude de Wittgenstein ${ }^{32}$. D'autre part, le cœur du diagnostic porté sur le mouvement illégitime d'universalisation du doute sceptique (de la mise ne cause d'une prétention particulière à sa mise en cause universelle) est contextualiste.

Dans l'appel au langage ordinaire, il ne faut pas pour autant lire un rejet des problèmes soulevés par la tradition moderne. Au contraire, pour Cavell, ce qui fait tout le prix des descriptions du second Wittgenstein, c'est que « le rejet du passé possède une nouvelle signification, comme s'il s'y ajoutait la conscience que l'histoire ne peut s'effacer sauf au prix de notre reconnaissance radicale de cette histoire, de notre reconnaissance en particulier qu'elle n'est pas du passé, que notre pratique et notre ambition propres ne peuvent être identifiées que contre la continuelle expérience du passé ${ }^{33}$ ». Vouloir dire ce que l'on dit devient alors affaire de rendre «son sens présent à soi-même ${ }^{34}$ ". L'arrogance de la voix qui par la description décide de nous reconduire aux usages ordinaires n'est autre que celle d'une autorité qui s'assume comme représentative et exemplaire de la condition humaine. Le dogmatisme qui résulte de cette autorité assumée et revendiquée ne s'arroge donc rien de plus que cette représentativité, ou plus exactement, le pari de sa possibilité.

31. Les Voix de la raison, op. cit., p. 88

32. Nous avons analysé ce déplacement du savoir à la certitude dans notre Introduction à De la certitude, Ellipses, 2006; et plus récemment dans notre contribution au dossier de la revue Théorèmes, " De la certitude religieuse: Wittgenstein sur la corde raide ", http://theoremes.revues.org/238.

33. Dire et vouloir dire, "Un public pour la philosophie ", op. cit., p. 61.

34. Ibid. 